Niedrig-Risiko-Prostatakarzinom

\title{
Active Surveillance wirkt sich positiv auf Lebensqualität aus
}

- Zum Langzeiterfolg der Active Surveillance in der Behandlung des nicht metastasierten Prostatakarzinoms gibt es bislang noch keine klinischen Daten, daher ist man bislang noch auf Computer-Simulationen angewiesen. Ein US-amerikanisches Forscherteam hat nun anhand einer fiktiven Kohorte von 65-jährigen Männern ein Modell aufgestellt, wie sich die Active Surveillance auf Lebensqualität und das Gesamtüberleben auswirkt [Hayes JH et al. JAMA 2010;304:2373-80]. Die Forscher setzten in ihrem Modell voraus, dass bei den Patienten ein Niedrig-Risiko-Prostatakarzinom (PSA-Wert < $10 \mathrm{mg} / \mathrm{ml}$, Gleason-Score $\leq 6$ ) diagnostiziert wurde. Als Behandlungsoption standen Brachytherapie, eine intensitätsmodulierte Strahlentherapie (IMRT), radikale Prostatektomie oder eben Active Surveillance zurVerfügung. Anhand einer Literaturauswertung wurde ermittelt, welchen Gesundheits- zustand die Patienten präferierten. Diese Zustände waren Grundlage für QALYs („quality-adjusted life-years"), also die Zeit, in welcher der Patient je nach Behandlungsmethode im gewünschten Gesundheitszustand verbringt.

Die effektivste Strategie war dabei mit 11,07 QALYs Active Surveillance mit anschließender IMRT bei Tumorprogression. Es folgten sofortige Brachytherapie (10,57 QALYs), IMRT (10,51 QALYs) und radikale Prostatektomie (10,23 QALYs). Selbst wenn man das erhöhte Sterberisiko berücksichtigt, hat die abwartende Haltung ein halbes Jahr Lebensqualität gegenüber der nächstbesten Option Brachytherapie zur Folge.

Die Forscher geben jedoch zu bedenken, dass ihr Modell auf zahlreichen Annahmen beruht und jeder Patient im Einzelfall anders entscheidet.

slx

\section{Riskante Praxis}

\section{Dosis von geteilten Tabletten kann stark variieren}

— Tabletten zu teilen, kann riskant sein, vor allem bei Medikamenten mit einem engen Bereich zwischen therapeutischer und toxischer Dosis. Denn je nach Tablettenart und Teilmethode kann es zu Dosisabweichungen kommen, wie belgische Wissenschaftler herausfanden.

Fünf Freiwillige im Alter zwischen 21 und 55 Jahren wurden aufgefordert, acht in der Größe und im Aussehen unterschiedliche Tabletten zu halbieren oder zu vierteln. Nur einer von ihnen hatte Erfahrungen im Tablettenteilen. Die Tabletten wurden mithilfe eines Tabletten-

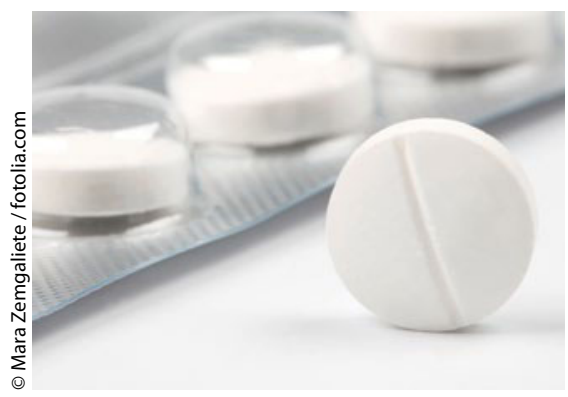

Wie genau lässt sich diese Tablette teilen? teilers (Gruppe 1), einer Schere, wenn die Tablette keine Kerbe hatte oder von Hand, wenn die Tablette eine Kerbe hatte (Gruppe 2) oder mit einem Küchenmesser (Gruppe 3) geteilt. Anschließend wurde das Gewicht der Tablettenstücke ermittelt: $31 \%$ wichen mehr als $15 \%$ von ihrem theoretischen Sollgewicht ab und $14 \%$ mehr als $25 \%$. Die genauesten Ergebnisse wurden mit dem Tablettenteiler erzielt. Trotzdem wichen auch hier noch $21 \%$ der Teilergebnisse mehr als $15 \%$ und $8 \%$ mehr als $25 \%$ vom zu erzielenden Soll ab. Einer deutschen Studie zufolge werden mehr als ein Viertel aller Tabletten geteilt, um die Dosisflexibilität zu erhöhen, damit die Tabletten besser zu schlucken sind oder um Kosten zu sparen (für die Patienten, aber auch für die Krankenkassen). Doch die Tablettenteile sind oft ungleich groß und es gehen - je nach Methode - auch mal Stücke verloren. Daran sollte man unbedingt denken. Die Studienautoren fordern zudem die Arzneimittelhersteller auf, in Zukunft ein breiteres Dosisspektrum der einzelnen Medikamente oder flüssige Alternativen anzubieten. ke

\section{Ehe hält gesund}

Verheiratete erhalten eher Spenderniere

— Heiraten ist offenbar gut für die Gesundheit - und verbessert für Dialysepatienten die Chancen auf eine Spenderniere. Das sind die Ergebnisse einer aktuelle Studie im „American Journal of Tansplantation“. Der Studie zufolge hatten verheiratete, geschiedene oder getrennt lebende Dialysepatienten eine mehr als $50 \%$ höhere Chance, auf der Warteliste für eine neue Niere zu landen als Singles. Bei Verheirateten war zudem die Wahrscheinlichkeit, dann tatsächlich auch ein Spenderorgan zu erhalten, um $28 \%$ höher als bei alleinstehenden Patienten auf der Warteliste.

Ein möglicher Grund für diese Ergebnisse sind der soziale Rückhalt und die Unterstützung im Alltag, die Dialysepatienten von ihren Ehepartnern während der aufwendigen Behandlung erfahren. Ein Partner an der Seite helfe einem, während der Dialyse "so gesund wie möglich" zu bleiben. Böte sich dann die Gelegenheit, ein Spenderorgan zu erhalten, seien verheiratete Dialysepatienten fit genug, um für die Transplantation infrage zu kommen. Dass sich Verheirateten diese Gelegenheit häufiger bietet als Singles, liegt laut den Studienautoren möglicherweise daran, dass Eheleute besser sozial vernetzt sind als Alleinstehende. Oft würde sich der gesunde Ehepartner für eine Lebendspende bereit erklären. Auch nach einer Scheidung sei die soziale Bindung an den Ex-Partner und dessen Freundeskreis noch so stark, dass die Bereitschaft zu einer Organspende vorhanden sei.

Heiraten, um sich für künftigen Bedarf eine Spenderniere zu sichern - diesen pauschalen Rat mögen die Studienautoren ihren Lesern aber dann doch nicht geben. Doch könne es nicht schaden, sich als Single einen großen Freundeskreis zuzulegen, sich gut zu vernetzen und generell auf seine Gesundheit zu achten.

men 